\title{
Phase pupil functions for reduction of defocus and spherical aberrations
}

\author{
Samir Mezouari and Andrew R. Harvey* \\ School of Engineering and Physical Sciences, Heriot-Watt University, Riccarton, Edinburgh EH14 4AS, UK
}

Received December 3, 2002

Radially symmetric pupil plane phase retardation functions are derived that extend focal depth and alleviate third-order spherical aberration (SA) effects. The radial symmetry of these functions means that they can be more conveniently manufactured by use of traditional techniques such as diamond machining than previously reported filters with rectangular symmetry. The method employs minimization of the variation of Strehl ratio with defocus, $W_{20}$, and SA, $W_{40}$. The performance of the derived phase filters is illustrated by comparison with standard optical systems and with previously reported phase filters. (c) 2003 Optical Society of America OCIS codes: $110.2990,050.1960$.

Several authors reported the use of amplitude filters to control defocus and reduce third-order aberrations such as Seidel's spherical aberration (SA) effects. ${ }^{1-6}$ However, phase filters may also be used, ${ }^{7-11}$ and they offer the advantage of improved optical efficiency. More recently, a novel approach that combines phase filters with digital processing to alleviate optical aberrations was reported. ${ }^{9,12,13}$ In this Letter we describe the design of such a phase filter, obtained from the evaluation of the Strehl ratio of circularly symmetric optical systems, the intensity distribution along the optical axis of which is evaluated by use of the stationary phase approximation. ${ }^{12}$ The performance of this phase filter in combination with an ideal lens is compared with that of an ideal lens without the phase filter and with previously reported phase filters that exhibited rectangular symmetry. The derived phase filters exhibit radial symmetry and can thus be easily manufactured.

The on-axis intensity distribution of an optical system that suffers from defocus of $W_{20}$ and SA of $W_{40}$, measured in units of wavelength, is given $b y^{2}$

$$
\begin{aligned}
I\left(W_{20}, W_{40}\right)= & 4 \pi^{2} \mid \int_{0}^{\infty} \tilde{p}(\rho) \exp \left\{i 2 \pi \left[W_{20}\left(\rho / \rho_{0}\right)^{2}\right.\right. \\
& \left.\left.+W_{40}\left(\rho / \rho_{0}\right)^{4}\right]\right\}\left.\rho \mathrm{d} \rho\right|^{2}
\end{aligned}
$$

where $\rho$ is the radial spatial frequency whose maximum value is the cutoff frequency, $\rho_{0} . \tilde{p}(\rho)$ represents the generalized pupil function, which for a radially symmetric pupil can be represented as

$$
\tilde{p}(\rho)= \begin{cases}\exp [i 2 \pi \theta(\rho)] & 0 \leq \rho \leq \rho_{0} \\ 0 & \rho>\rho_{0}\end{cases}
$$

where $\theta(\rho)$ denotes the pupil phase function.

Inserting Eq. (2) into Eq. (1) with a change of variable enables us to rewrite Eq. (1) in a convenient form:

$$
\begin{aligned}
I\left(W_{20}, W_{40}\right)= & \pi^{2} \rho_{0}{ }^{4} \mid \int_{-\infty}^{\infty} \exp \left\{i 2 \pi \left[\Phi(\xi)+W_{40} \xi^{2}\right.\right. \\
& \left.\left.+\left(W_{20}+W_{40}\right) \xi\right]\right\}\left.\mathrm{d} \xi\right|^{2} \\
& 0146-9592 / 03 / 100771-03 \$ 15.00 / 0
\end{aligned}
$$

where $\xi=\left(\rho / \rho_{0}\right)^{2}-1 / 2$ and $\Phi(\xi)=\theta(\rho)$. The pupil function is nonzero for $0 \leq \rho \leq \rho_{0}$, corresponding to $-1 / 2 \leq \xi \leq 1 / 2$. Equation (3) describes the variation of the on-axis intensity with defocus and SA. The aim of this work is to find an expression for the pupil phase function $\Phi(\xi)$, which results in an irradiation distribution that is insensitive to defocus and SA. It will be shown that, although the design process reduces the variation of only the axial intensity, a reduction in sensitivity to aberration of the complete point-spread function (PSF) is nevertheless obtained. We employ the stationary phase approximation to evaluate Eq. (3), where stationary points are given by

$$
\left.\frac{\mathrm{d}}{\mathrm{d} \xi}\left[\Phi(\xi)+W_{40} \xi^{2}+\left(W_{20}+W_{40}\right) \xi\right]\right|_{\xi=\xi_{s}}=0,
$$

and $\xi$ denotes the stationary point. The evaluation of the axial irradiation distribution, as given by Eq. (3), with the stationary phase approximation, ${ }^{14}$ is given by

$$
I\left(W_{20}, W_{40}\right) \cong 2 \pi^{3} \rho_{0}{ }^{4}\left|\frac{1}{2 W_{40}+\Phi^{\prime \prime}\left(\xi_{s}\right)}\right|,
$$

where $\Phi^{\prime \prime}$ represents the second derivative of $\Phi$. The independence of the axial irradiation distribution on defocus aberration and SA leads to the following equations:

$$
\begin{aligned}
& \frac{\mathrm{d}}{\mathrm{d} W_{20}}\left|\frac{1}{2 W_{40}+\Phi^{\prime \prime}\left(\xi_{s}\right)}\right|=0, \\
& \frac{\mathrm{d}}{\mathrm{d} W_{40}}\left|\frac{1}{2 W_{40}+\Phi^{\prime \prime}\left(\xi_{s}\right)}\right|=0 .
\end{aligned}
$$

There is no rigorous mathematical solution that makes possible simultaneous control of both defocus and SA. In contrast, solutions exist that permit the design of phase filters that reduce either defocus aberration only [by resolving Eqs. (4) and (6a)] or SA only [by resolving Eqs. (4) and (6b) together]. Therefore, we deduce the phase filter function that controls the SA when the optical system is in the best focal plane $\left(W_{20}=0\right)$ by integrating the differential equation system (4) and (6b) twice. For brevity, the details of the calculation are not given here. The resulting phase function enables the pupil phase function to be 
written as

$$
\theta(\rho)=\beta_{0}\left(\rho / \rho_{0}\right)^{4}+\beta\left(\rho / \rho_{0}\right)^{4} \log \left(\rho / \rho_{0}\right),
$$

where the coefficients $\beta_{0}$ and $\beta \neq 0$ are real. The first quartic term in Eq. (7) represents a SA shift, and therefore the sensitivity of the on-axis intensity to SA is controlled by the coefficient $\beta$. One can employ the circularly symmetric phase filter described by Eq. (7) to alleviate the SA when the optical system is well focused. A similar approach was recently reported in Ref. 15, in which the authors derived axicons that are illuminated with a Gaussian Schell model.

An example of the axial variation of SA with the filter described by Eq. (7) is shown in Fig. 1. Tolerance to SA is enhanced approximately seven times, according to Rayleigh's criterion for aberration peak value, which is equivalent to a normalized intensity of 0.8 . Moreover, as shown in Fig. 2, the tolerance to defocus, on axis, is improved by a factor of 2 in comparison with an ideal circular lens. As discussed below, there is an increase in the width of the PSF with a consequent reduction in axial intensity, as indicated by the scaling parameters in the figure. Logarithmic filters (LFs) were previously reported ${ }^{8,13}$; however, contrary to our approach, the derivation of such filters was mainly achieved from geometric-optics considerations without including diffraction effects.

As mentioned above, the phase functions that reduce the defocus error when the SA is negligible $\left(W_{40} \approx 0\right)$ are obtained by combination of Eqs. (5) and (7a) to yield $\Phi(\xi)=\alpha \xi^{2}$, and hence

$$
\theta(\rho)=\alpha\left(\left(\rho / \rho_{0}\right)^{2}-1 / 2\right)^{2},
$$

where the coefficient $\alpha \neq 0$ is a constant. A linear term in Eq. (8) that represents a simple focal shift is neglected. A similar result was also obtained by Zalvidea and Sicre $^{10}$ by exploring the properties of a Wigner distribution function. The quartic filter $(\mathrm{QF})$ in Eq. (8) displays the same performance, on axis, as the axilens $\mathrm{LF}^{8}$ and differs only by a focal shift. ${ }^{10} \mathrm{~A}$ more comprehensive comparison is given in Ref. 11. As was demonstrated in Ref. 10, the QF [as given in Eq. (8)] achieves the best tolerance to defocus at $\alpha=0.75 \pi$. Reduced sensitivity of on-axis intensity for SA and defocus are also shown in Figs. 1 and 2, respectively.

We derived the phase filters by requiring only that the axial intensity be insensitive to aberration, and we show here through calculation of the resulting modulation transfer function (MTF) that the transverse PSF is also insensitive to defocus and SA. The MTFs are shown in Fig. 3 for up to two wavelengths of defocus and SA for an ideal lens, the $\mathrm{QF}$, and radial logarithmic and rectangularly separable LFs. It can be seen that all three filters reduce sensitivity to defocus and $\mathrm{SA}$ with respect to an ideal lens but with a reduction in the MTF. Both logarithmic phase filters give less variation of the MTF with defocus and SA than the $Q F$, although the reduced MTF at high spatial frequencies is more pronounced in the presence of defocus with the radially symmetric LF (the effective cutoff frequency is $65 \%$ of the diffraction-limited case) than the quartic or rectangularly symmetric LFs. Conversely, the $\mathrm{QF}$ has a more compact PSF but is more sensitive to defocus and SA. All filters increase the tolerance to moderate amounts of SA; the $\mathrm{QF}$ is superior for small $\mathrm{SA}$, and the linearly separable LF is superior for larger amounts of SA.

It is particularly pertinent to compare the curves in Fig. 3 for the MTFs of the radially symmetric LF with the MTFs of the rectangularly separable LF. ${ }^{12}$ The effect inherent in the use of all phase filters is to give MTFs that are significantly lower than those of an ideal lens but are much less sensitive to aberration and contain no zeros. The absence of zeros in the MTF means that digital restoration of the recorded image is possible. Thus, although the reduction in MTF corresponds to a broadening in PSF, digital restoration will permit an almost diffraction-limited PSF to be restored, whereas this is not possible for the simple aberrated lens. Because the magnitude of the resulting MTFs is reduced, deterioration in the signal-to-noise ratio is expected in the restored image. ${ }^{16}$ The relative constancy of the MTF enables a single deconvolution kernel to be used without accurate knowledge of the optical aberration.

For the radially symmetric phase filter derived here, reduced sensitivity to aberrations can be achieved by an increase the value of the parameter $\beta$; however, this is accompanied by a rapid reduction of the on-axis intensity, as shown in Fig. 4. There is also a

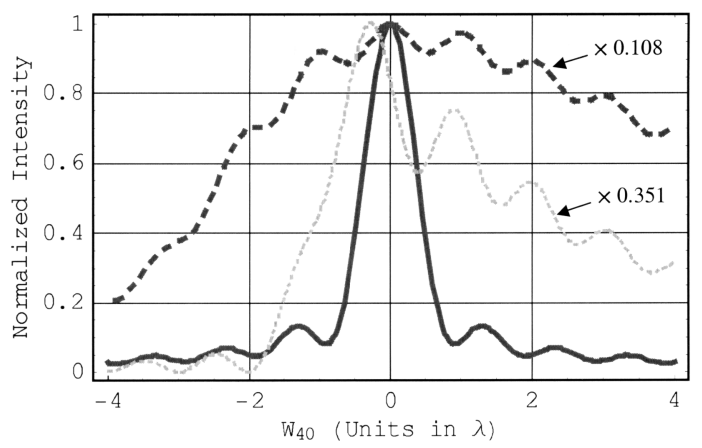

Fig. 1. Axial intensity as a function of SA of $W_{40}$, with zero-defocus aberration. The solid curve represents an ideal circular lens, the dark dashed plot corresponds to the use of an additional $\operatorname{LF}\left(\beta=5.6 \pi\right.$ and $\left.\beta_{0} / \beta=0.401\right)$, and the light dotted plot corresponds to the QF $(\alpha=0.75 \pi)$.

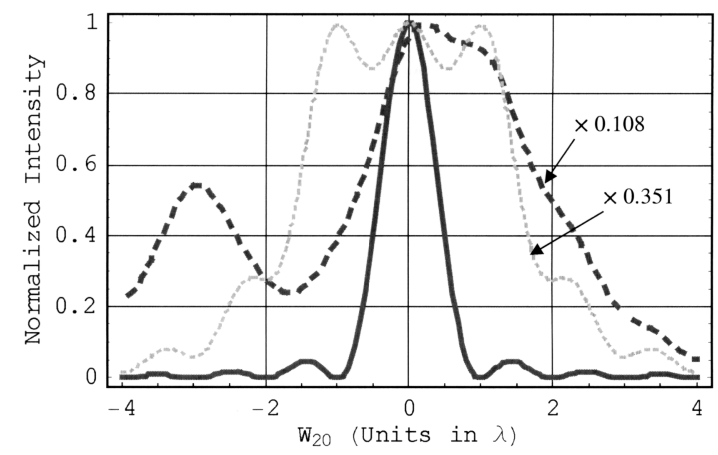

Fig. 2. Axial intensity as a function of defocus of $W_{20}$. The curves are as defined in Fig. 1. 


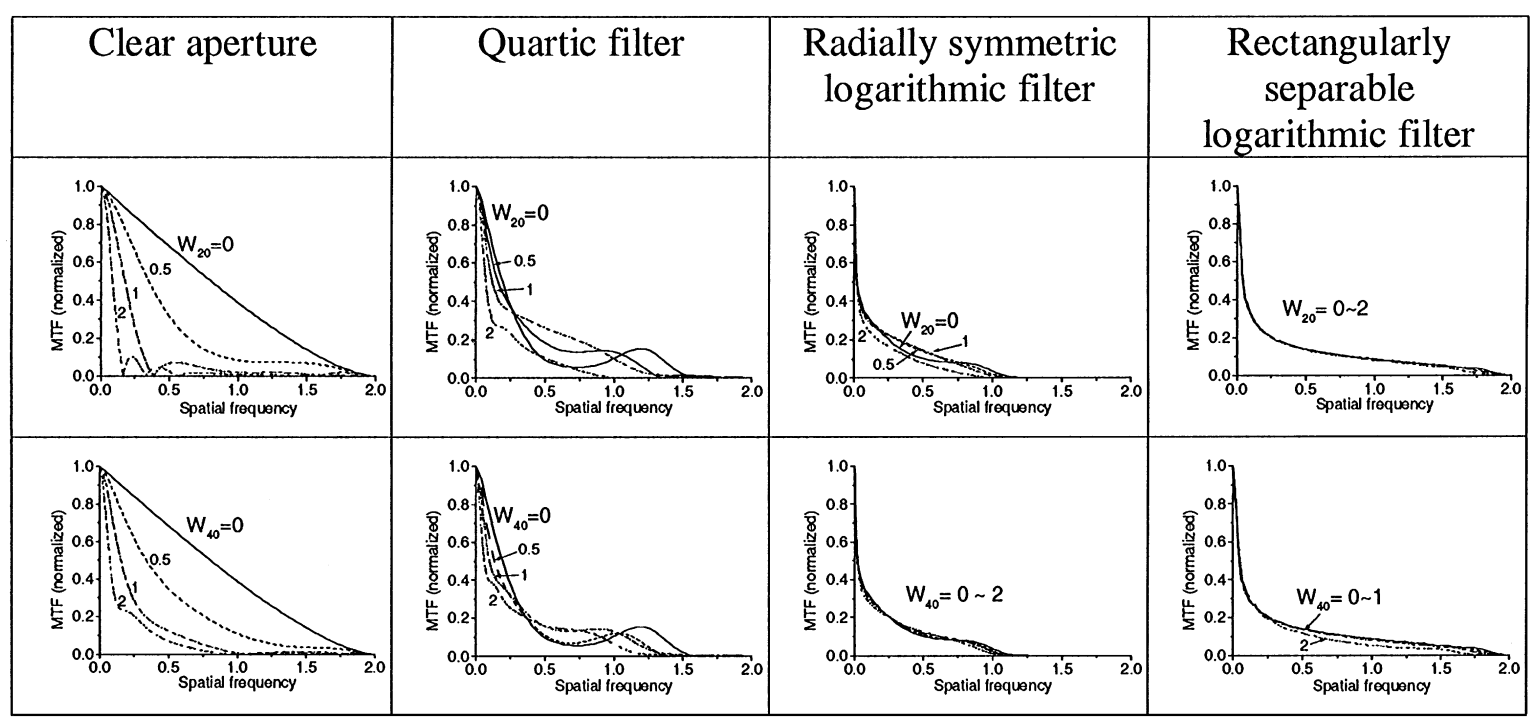

Fig. 3. Computed MTFs obtained with optical systems suffering from defocus $W_{20}$ and spherical aberration $W_{40}$ of $0, \lambda / 2$, $\lambda$, and $2 \lambda$. The rectangularly separable filter has a $4 \pi$ peak-to-valley optical path difference.

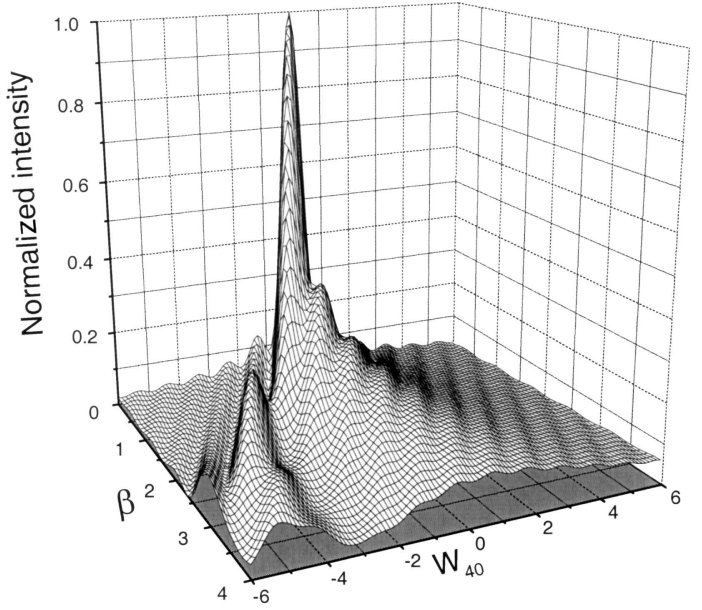

Fig. 4. Variation of the intensity of the focal point as a function of the SA parameter, $W_{40}$, and $\beta$. When $\beta=0$, the pupil function is identical to a circular aperture, and the maximum intensity (aberration free, $W_{40}=0$ ) is normalized to 1 . The relative intensity decreases drastically as $\beta$ increases.

considerable decrease in the magnitude of the MTF overall, particularly at high spatial frequencies, and this increase will reduce the signal-to-noise ratio and the effective resolution of the restored image. Although the use of the radially symmetric LF provides significant scope for improving the imaging performance of aberrated optical systems, this reduction in MTF with increasing $\beta$ places a practical limit on the achievable insensitivity to aberration.

In summary, we have presented a simple method to design radially symmetric phase-only filters that exhibit high tolerance to defocus and SA. When this method is used in imaging systems subject to SA and defocus, significant improvements in imaging performance can be obtained. From a purely theoretical point of view the radially symmetric phase filters appear to offer performance inferior to that of the rectangularly separable LF; however, the radial symmetry means that these filters are more readily manufactured at low cost. For example, a major potential application for this technology is in athermalization and achromatization of thermal imaging lenses, the radial symmetry of which permits manufacture at reduced cost by diamond machining.

This work was carried out with funding from the Sensors and Electronic Warfare Research Domain of the UK Ministry of Defence Corporate Research Programme.

*Principal contact; e-mail a.r.harvey@hw.ac.uk.

\section{References}

1. W. T. Welford, J. Opt. Soc. Am. 50, 749 (1960).

2. J. Ojeda-Castañeda, P. Andrés, and A. Díaz, Opt. Lett. 11, 487 (1986).

3. J. Ojeda-Castaneda and L. R. Berriel Valdos, Opt. Lett. 13, 183 (1988).

4. J. Ojeda-Castaneda, L. R. Berriel-Valdos, and E. L. Montes, Opt. Lett. 10, 520 (1985).

5. M. Mino and Y. Okano, Appl. Opt. 10, 2219 (1971).

6. J. Ojeda-Castaneda and L. R. Berriel-Valdos, Appl. Opt. 29, 994 (1991).

7. N. Davidson, A. A. Friesem, and E. Hasman, Opt. Lett. 16, 523 (1991).

8. J. Sochacki, S. Bara, Z. Jaroszewicz, and A. Kolodziejczyk, Opt. Lett. 17, 7 (1992).

9. E. R. Dowski, Jr., and W. T. Cathey, Appl. Opt. 34, 1859 (1995).

10. D. Zalvidea and E. E. Sicre, Appl. Opt. 37, 3623 (1998).

11. D. Zalvidea, C. Colautti, and E. E. Sicre, J. Opt. Soc. Am. A 17, 867 (2000).

12. S. S. Sherif, E. R. Dowski, and W. T. Cathey, Proc. SPIE 4471, 272 (2001).

13. W. Chi and N. George, Opt. Lett. 26, 875 (2001).

14. V. A. Borovikov, Uniform Stationary Phase Method, Vol. 40 of IEE Electromagnetic Waves Series (Institution of Electrical Engineers, London, 1994).

15. A. Thaning, A. T. Friberg, S. Y. Popov, and Z. Jaroszewicz, J. Opt. Soc. Am. A 19, 491 (2002).

16. S. Mezouari and A. R. Harvey, Proc. SPIE 4768, 21 (2002). 